\title{
A e-democracia sanitária no Brasil: em busca da identificação de atores de mecanismos virtuais de participação na elaboração de normas de direito sanitário
}

\section{E-democracy health in Brazil: searching for identifying participants in the development of health law}

\author{
Sueli Galdolfi Dallari \\ Universidade de São Paulo. Faculdade de Saúde Pública. Centro \\ de Estudos e Pesquisas de Direito Sanitário. São Paulo, SP, Brasil. \\ E-mail: sdallariळusp.br

\section{Fernando Mussa Abujamra Aith} \\ Universidade de São Paulo. Faculdade de Medicina. Centro de \\ Estudos e Pesquisas de Direito Sanitário. São Paulo, SP, Brasil. \\ E-mail: fernando.aithळusp.br

\section{Deisy de Freitas Lima Ventura} \\ Universidade de São Paulo. Instituto de Relações Internacionais. \\ Faculdade de Saúde Pública. Centro de Estudos e Pesquisas de \\ Direito Sanitário. São Paulo, SP, Brasil. \\ E-mail: deisy.venturaळusp.br

\section{Lúcia Dias da Silva Guerra} \\ Universidade de São Paulo. Faculdade de Saúde Pública. Centro \\ de Estudos e Pesquisas de Direito Sanitário. São Paulo, SP, Brasil. \\ E-mail: ludsguerraळgmail.com
}

\section{Renata Rothbarth Silva}

Universidade de São Paulo. Faculdade de Saúde Pública. Centro de Estudos e Pesquisas de Direito Sanitário. São Paulo, SP, Brasil. E-mail: rothbarthळusp.br

\section{Matheus Zuliane Falcão}

Universidade de São Paulo. Faculdade de Direito. Centro de Estudos e Pesquisas de Direito Sanitário. São Paulo, SP, Brasil.

E-mail: mzfalcoœgmail.com

\section{Yasmin Bujdoso}

Universidade de São Paulo. Faculdade de Saúde Pública. Centro de Estudos e Pesquisas de Direito Sanitário. São Paulo, SP, Brasil. E-mail: yasminlilla®yahoo.com.br

\section{Rachelle Amália Agostini Balbinot}

Universidade de São Paulo. Faculdade de Saúde Pública. Centro de Estudos e Pesquisas de Direito Sanitário. São Paulo, SP, Brasil. E-mail: rachebalbi®gmail.com

\section{Correspondência}

Sueli Galdolfi Dallari

Universidade de São Paulo. Faculdade de Saúde Pública. Centro de Estudos e Pesquisas de Direito Sanitário. Avenida Dr. Arnaldo,

715, Subsolo, SII. São Paulo, SP, Brasil. CEP 01246-904

\section{Resumo}

Esta comunicação oferece os resultados da primeira fase da pesquisa franco-brasileira $A$ e-democracia sanitária na França e no Brasil: impacto sobre a evolução do direito da saúde. No âmbito desta pesquisa, democracia sanitária é um processo de reflexão, de concertação e de decisão previsto ou reivindicado para a elaboração de uma política de saúde; e "e-Democracia sanitária" é o seu exercício quando mediado pelas tecnologias de informação e comunicação. Entre os objetivos do projeto encontra-se a identificação empírica dos atores da e-democracia sanitária no Brasil. Foram analisados relatórios de consultas públicas promovidas pela Agência Nacional de Saúde Suplementar, pela Agência Nacional de Vigilância Sanitária, pela Comissão Nacional de Incorporação de Tecnologias no SUS e pelo Ministério da Saúde no ano de 2014. Como conclusões preliminares, emergem a constatação de um déficit significativo de transparência em relação aos mecanismos virtuais de consulta estudados e a necessidade de padronização dos dados apresentados em seus respectivos relatórios.

Palavras-chave: Democracia; Participação Cidadã; Internet; Direito à Saúde; Comunicação em Saúde. 


\section{Abstract}

This Communication provides the results of the first phase of the Franco-Brazilian research $\varepsilon$-democracy health in France and Brazil: impact on the evolution of the health law. Within this research, health democracy is a process of reflection, dialogue and decision-making plans and it is claimed for providing health policies; in fact, health e-Democracy is a health policy exercise when mediated by information and communication technologies. The objective of this study is to identify participants that were involved in the health e-democracy in Brazil. We analyzed public consultation reports promoted by the National Agency of Supplemental Health, the National Health Surveillance Agency, the National Commission for Technology Incorporation under Unified Health System and the Ministry of Health in the year of 2014. As preliminary conclusions we point out a significant deficit of transparency regarding those studied virtual mechanisms and the need for standardization of data presented in its respective reports.

Keywords: Democracy; Citizen Participation; Internet; Right To Health; Health Communication.

\section{Introdução}

Esta comunicação oferece os resultados da primeira fase da pesquisa franco-brasileira $A e$ - $d e$ mocracia sanitária na França e no Brasil: impacto sobre a evolução do direito da saúde. De uso corrente na França, a expressão "democracia sanitária” é polissêmica e ainda pouco referida no Brasil. No âmbito desta pesquisa, ela significa um processo de reflexão, de concertação e de decisão previsto ou reivindicado para a elaboração de uma política de saúde (Tabuteau, 2013). Logo, implica a participação das pessoas, em graus diversos, na tomada de decisões que referem-se à sua vida, à defesa dos direitos humanos e da igualdade de todos diante dos sistemas de saúde, e ao direito dos pacientes e de seus acompanhantes de expressar sua opinião e o seu consentimento (Demailly, 2014).

No Brasil, em particular, a democracia sanitária pode ser um caminho para que a tensão entre os Poderes Executivo e Legislativo na produção normativa se transforme em pacto social em prol da proteção do direito à saúde, tendo como base a criação de mecanismos de participação da sociedade (Aith; Dallari, 2014). Entre estes mecanismos, prosperam as iniciativas digitais (Mendonça; Amaral, 2014) impulsionadas pelo Estado, por organizações civis, partidos, empresas e indivíduos, valendo-se de práticas bastante diversas - por exemplo, consultas públicas online, plataformas de produção colaborativa de conhecimento, chats com autoridades, blogs e grupos de discussão em redes sociais. Numerosas expressões estão associadas à produção de conhecimento e ao debate brasileiro sobre esta temática, com claro predomínio dos termos democracia digital e democracia virtual (Gomes, 2010), secundados por expressões como ciberdemocracia, democracia eletrônica, governo eletrônico, e-democracia etc. $\mathrm{Na}$ literatura especializada em saúde, as expressões inclusão digital e inclusão informacional apontam que não basta garantir o acesso à informação pelos atores sociais; é necessário haver a apropriação de seu significado, pertinência e relevância em relação ao objetivo que se pretende (Moraes et al., 2009).

$\mathrm{Na}$ impossibilidade de desenvolver nesta breve comunicação um debate conceitual, optamos 
simplesmente por denominar "e-Democracia sanitária" como o exercício da democracia sanitária que é mediado pelas tecnologias de informação e comunicação.

O principal interesse deste estudo comparativo entre o Brasil e a França é o contraste entre a escassa institucionalização da participação social francesa, a que corresponde um intenso ativismo social extrainstitucional, inclusive por meio da internet; e a grande institucionalização da participação social brasileira, inclusive por meio da internet, que aparentemente tende a reduzir o ativismo virtual extrainstitucional. A aprendizagem recíproca destas experiências poderia resultar na formulação de sugestões para o aperfeiçoamento dos mecanismos de participação, eis que a escolha de um formato adequado pode ser uma das variáveis fundamentais para a continuidade das experiências participativas (Avritzer, 2008).

Entre os objetivos do projeto encontra a identificação empírica dos suportes e das modalidades de participação virtual nos processos decisórios de saúde na França e no Brasil, a começar por seus atores, que é o objeto desta comunicação. Em suas etapas futuras, a pesquisa buscará determinar se e como a internet tornou-se uma fonte de direito no Brasil e na França, a fim de investigar, por meio de pesquisa qualitativa e estudos de caso, o quanto as entidades regulatórias apreendem das contribuições apresentadas pelos atores sociais pela via destes mecanismos, cotejando a participação no processo de elaboração e a versão final de algumas normas selecionadas.

\section{Metodologia}

Classificamos os mecanismos virtuais de participação como ascendentes ou descendentes. Mecanismos virtuais ascendentes é a forma de participação proveniente da sociedade, por meio de diversas manifestações individuais ou coletivas, instando o Estado a adotar uma norma ou dar efetividade a uma norma preexistente. Mecanismos virtuais descendentes, por sua vez, é a forma de participação promovida por órgãos e instituições estatais por meio de mecanismos virtuais, a fim de ampliar o debate sobre temas que figuram na pauta política dos governos. Os resultados aqui apresentados concernem exclusivamente à democracia descendente no Brasil.

Como amostra de atores de democracia descendente, foram identificados por meio do canal eletrônico Portal Brasil os órgãos federais que promovem consultas públicas durante a elaboração de normas de saúde, quais sejam: agências reguladoras, ministérios, conselhos, fundações, autarquias e empresas públicas. A partir desta lista, selecionamos as instituições que utilizam com maior regularidade o instrumento das consultas públicas em seus processos decisórios: Agência Nacional de Saúde Suplementar (ANS), Agência Nacional de Vigilância Sanitária (Anvisa), Comissão Nacional de Incorporação de Tecnologias no SUS, Ministério da Saúde (MS) e Instituto Nacional de Propriedade Industrial (Inpi). Nos sites destes órgãos, foram identificadas as consultas públicas realizadas por meio de mecanismos virtuais e obtidos os relatórios concernentes a algumas delas.

Foram analisados todos os relatórios disponíveis na busca da identificação dos atores que apresentaram contribuições. 0 recorte temporal adotado no âmbito do presente artigo foi o ano de 2014, o que explica a ausência do Inpi dos primeiros resultados por não ter promovido consultas naquele período. Os sites dos órgãos selecionados foram consultados no período de maio a junho de 2015.

\section{Resultados e discussão}

A primeira dificuldade enfrentada na realização desta pesquisa foi escassez de informação sobre as consultas públicas realizadas pelos órgãos selecionados, eis que das 168 consultas promovidas, apenas 58 relatórios foram publicados. Isto significa que os resultados aqui apresentados não são exaustivos, no sentido de que eles baseiam-se apenas nas consultas sobre as quais a informação é disponível, e não sobre o total de consultas realizadas, cobrindo, portanto, 34,53\% do número total de consultas. Não foram identificados, na informação oficial, critérios que justifiquem a publicação ou não de relatórios sobre as consultas públicas. 
No Gráfico 1, apresentamos o número de consultas públicas realizadas no ano de 2014 pelos órgãos selecionados, indicando com asterisco as consultas cujo relatório encontra-se disponível. Se considerarmos que a transparência pode ser aferida pela relação entre número de consultas realizadas e número de consultas cujos relatórios são disponíveis, entre os órgãos estudados a ANS seria a mais transparente (100\% das consultas relatadas), seguida do Conitec (82,75\%) e da Anvisa (27,7\%), enquanto o MS seria o menos transparente (apenas $3,57 \%$ dos relatórios encontram-se disponíveis). No entanto, este critério de aferição de transparência parece precipitado, eis que a prática das consultas públicas e especificamente a da elaboração de relatórios de participação social são muito recentes no Brasil. No futuro, porém, a aferição deste indicador pode vir a ser uma variável relevante.

Em relação à contribuição dos atores sociais, o Gráfico 2 revela significativo número de manifestações individuais e coletivas que, no conjunto dos órgãos selecionados, alcançam a cifra de 8.702. A Anvisa foi o órgão que recebeu o maior número de manifestações, como demonstra o Gráfico 2. Isto poderia ser explicado, entre outras razões, pelas ações específicas de encorajamento à participação, promovidas pela agência (BRASIL, 2011). No entanto, a principal característica destas contribuições é sua heterogeneidade, o que dificulta a formulação de hipóteses explicativas.

As informações fornecidas sobre estas manifestações, por sua vez, são também heterogêneas, o que dificultou nossa tarefa de identificação dos atores. Salvo no caso da Anvisa, os relatórios das demais entidades não informam como seus dados foram construídos. Na ausência de padronização dos dados obtidos, construímos cinco categorias de atores a partir das diversas classificações fornecidas pelos relatórios: cidadão; instituições públicas municipais, estaduais, federais; profissional de saúde; prestador/fornecedores; outro/sem identificação. Os atores que fazem parte das nossas categorias estão identificados na Tabela 1, assim como os resultados da pesquisa.

Nota-se o predomínio da participação da cidadania nos mecanismos de consulta promovidos pela Anvisa e pelo Conitec, enquanto os prestadores/fornecedores são predominantes nas consultas da ANS e os profissionais da saúde são ligeiramente mais numerosos do que os prestadores/fornecedores e as instituições públicas nas consultas do MS. Fica evidenciado, ainda, que a participação da cidadania é bastante inferior à das demais categorias na ANS e no MS.

Como conclusões preliminares desta pesquisa, emergem a constatação de um déficit significativo de transparência em relação aos mecanismos virtuais de consulta estudados, e a necessidade de construção de uma metodologia comum pelas entidades que adotam estas práticas para apresentação de informações sobre as consultas, sob pena de desencorajar os estudos sobre a e-democracia sanitária ou comprometer a fidedignidade dos seus resultados.

Na próxima etapa da pesquisa, a partir da identificação dos atores, buscaremos compreender a dinâmica da participação por meio dos mecanismos virtuais oferecidos pelas entidades estudadas, investigando, entre outros aspectos, os motivos pelos quais os atores participam, o eventual recurso a formas alternativas como a influência dos recursos financeiros e humanos disponíveis na capacidade de participação, as expectativas de acolhimento das demandas; buscando identificar o impacto que a participação via internet possui sobre o sistema de saúde, principalmente na jurisprudência e na produção normativa do direito sanitário no Brasil. 
Tabela I - Distribuição em categorias dos atores que participaram em consultas públicas realizadas pela Agência Nacional de Vigilância Sanitária, Agência Nacional de Saúde, Ministério da Saúde, Comissão Nacional de Incorporação de Tecnologias do SUS, ano 2014 (exceto a consulta n 54/2014 da ANS).

\begin{tabular}{|c|c|c|c|c|c|c|c|c|}
\hline \multirow{2}{*}{$\begin{array}{l}\text { Categoria da } \\
\text { pesquisa }\end{array}$} & \multicolumn{2}{|l|}{ ANVISA } & \multicolumn{2}{|l|}{ ANS } & \multicolumn{2}{|l|}{ MS } & \multicolumn{2}{|l|}{ CONITEC } \\
\hline & Composição & $\%$ & Composição & $\%$ & Composição & $\%$ & Composição & $\%$ \\
\hline Cidadão & $\begin{array}{l}\text { Cidadão, } \\
\text { cidadão ou } \\
\text { consumidor, } \\
\text { entidade de } \\
\text { defesa do } \\
\text { consumidor } \\
\text { ou associações } \\
\text { de pacientes, } \\
\text { sociedade civil } \\
\text { organizada }\end{array}$ & 65,38 & Consumidores & 4 & Pessoa física & 5,08 & $\begin{array}{l}\text { Associação } \\
\text { de pacientes, } \\
\text { individual }\end{array}$ & 31,18 \\
\hline Governo & $\begin{array}{l}\text { Órgão ou } \\
\text { entidade do } \\
\text { poder público, } \\
\text { outro órgão ou } \\
\text { entidade do } \\
\text { governo federal/ } \\
\text { estadual/ } \\
\text { municipal ou } \\
\text { do Sistema } \\
\text { de Vigilância } \\
\text { Sanitária }\end{array}$ & 1,77 & Gestor & 20 & $\begin{array}{l}\text { Instituição } \\
\text { pública }\end{array}$ & 15,25 & $\begin{array}{l}\text { Secretaria } \\
\text { Municipal, } \\
\text { Secretaria } \\
\text { de Estado, } \\
\text { Ministério } \\
\text { da Saúde, } \\
\text { instituição de } \\
\text { saúde }\end{array}$ & 23,92 \\
\hline $\begin{array}{l}\text { Profissional } \\
\text { de saúde }\end{array}$ & $\begin{array}{l}\text { Profissional de } \\
\text { saúde, outro } \\
\text { profissional } \\
\text { relacionado } \\
\text { ao tema, } \\
\text { investigador, } \\
\text { pesquisador } \\
\text { ou membro da } \\
\text { comunidade } \\
\text { científica, } \\
\text { academia ou } \\
\text { instituição } \\
\text { de ensino e } \\
\text { pesquisa pública } \\
\text { ou privada, } \\
\text { entidade de } \\
\text { classe ou } \\
\text { categoria } \\
\text { profissional de } \\
\text { saúde, conselho, } \\
\text { sindicato ou } \\
\text { associações } \\
\text { profissionais }\end{array}$ & 24,89 & $\begin{array}{l}\text { Universidades } \\
\text { federais e } \\
\text { estaduais, } \\
\text { associações } \\
\text { profissionais, } \\
\text { cooperativas, } \\
\text { federações } \\
\text { profissionais }\end{array}$ & 16 & Associação & 39 & $\begin{array}{l}\text { Profissionais, } \\
\text { instituição } \\
\text { de ensino, } \\
\text { sociedade } \\
\text { médica }\end{array}$ & 31,10 \\
\hline
\end{tabular}


Tabela I - Continuação

\begin{tabular}{|c|c|c|c|c|c|c|c|c|}
\hline \multirow{2}{*}{$\begin{array}{l}\text { Categoria da } \\
\text { pesquisa }\end{array}$} & \multicolumn{2}{|l|}{ ANVISA } & \multicolumn{2}{|l|}{ ANS } & \multicolumn{2}{|l|}{ MS } & \multicolumn{2}{|l|}{ CONITEC } \\
\hline & Composição & $\%$ & Composição & $\%$ & Composição & $\%$ & Composição & $\%$ \\
\hline $\begin{array}{l}\text { Prestador/ } \\
\text { fornecedores }\end{array}$ & $\begin{array}{l}\text { Empresa } \\
\text { privada, setor } \\
\text { - empresa } \\
\text { ou entidade } \\
\text { representativa }\end{array}$ & 6,55 & $\begin{array}{l}\text { Prestadoras, } \\
\text { operadoras }\end{array}$ & 54 & Empresa & 35,59 & Empresa & 3,84 \\
\hline $\begin{array}{l}\text { Outros/sem } \\
\text { identificação }\end{array}$ & Outros & 1,41 & $\begin{array}{l}\text { Demais } \\
\text { representantes } \\
\text { do Setor Público } \\
\text { ou Privado } \\
\text { (pessoa física ou } \\
\text { jurídica) }\end{array}$ & 6 & Outra & 5,08 & $\begin{array}{l}\text { Outro, sem } \\
\text { identificação }\end{array}$ & 9,96 \\
\hline
\end{tabular}

Gráfico I - Número de consultas realizadas e consultas com relatório disponível na Agência Nacional de Vigilância Sanitária, Agência Nacional de Saúde, Ministério da Saúde, Comissão Nacional de Incorporação de Tecnologias do SUS, ano 2014.

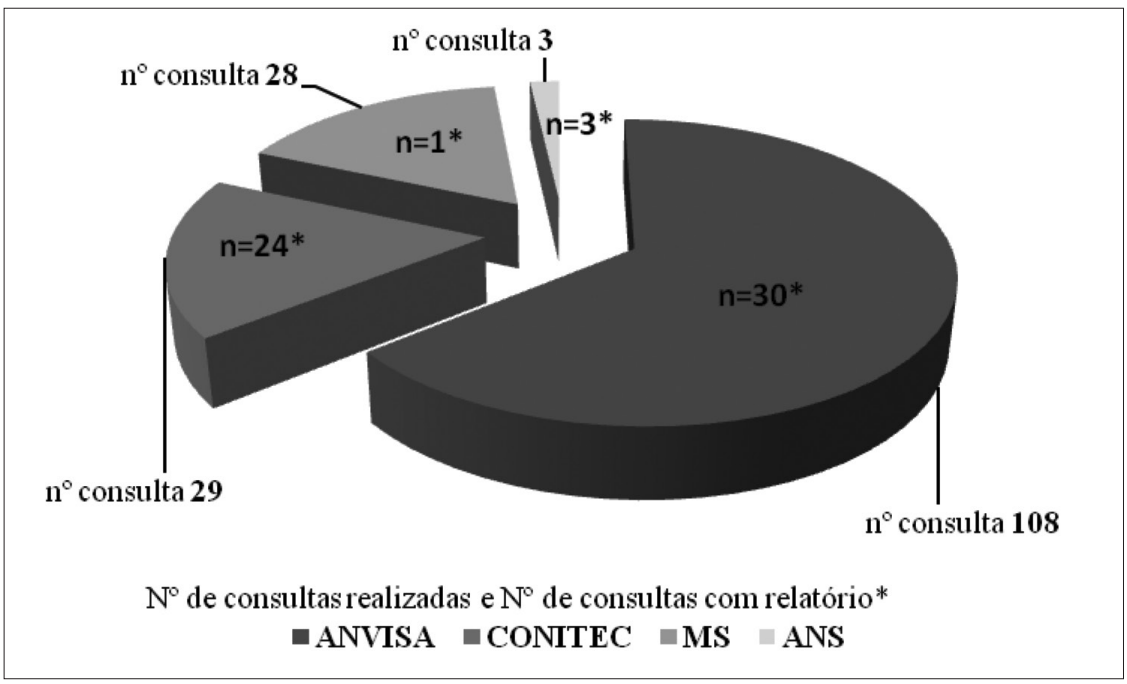


Gráfico 2 - Número de contribuições recebidas em consultas públicas segundo as consultas públicas com relatório, Agência Nacional de Vigilância Sanitária, Agência Nacional de Saúde, Ministério da Saúde, Comissão Nacional de Incorporação de Tecnologias do SUS, ano 2014.

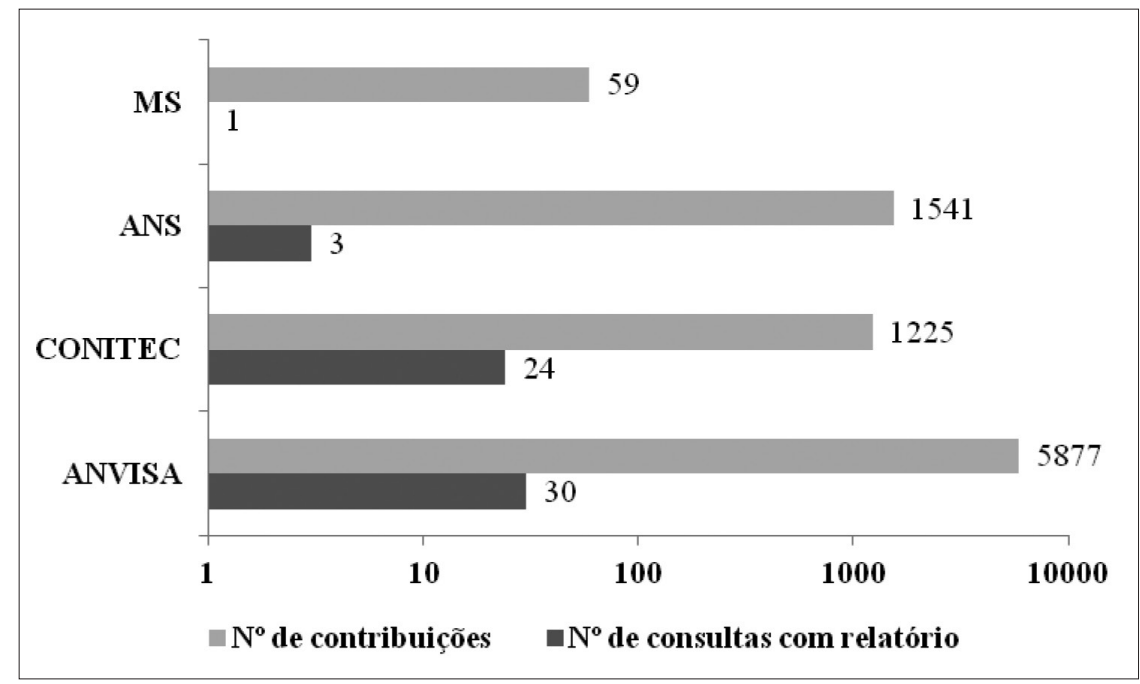

\section{Referências}

AITH, F. M. A.; DALLARI, S. G. Produção de normas jurídicas sobre saúde no âmbito do estado democrático de direito brasileiro. Cadernos de Saúde Pública, Rio de Janeiro, v. 3o, n. 10, p. 1-3, 2014.

AVRITZER, L. Instituições participativas e desenho institucional: algumas considerações sobre a variação da participação no Brasil democrático. Opinião Pública, São Paulo, v. 14, n. 1, p. 43-64, 2008.

BRASIL. Ministério da Saúde. Agência Nacional de Vigilância Sanitária. Instituto Brasileiro de Defesa do Consumidor. Projeto Fortalecimento da Capacidade Técnica para Participação Social na Regulação ANVISA/IDEC. Relatório final, Brasília, DF, maio 2011.

DEMAILLY, L. Variations de la démocratie sanitaire et politique publique de santé mentale en
France. Toulouse: SociologieS, 2014. Disponível em: <http://sociologies.revues.org/4653>. Acesso em: 28 abr. 2015 .

GOMES, W. Democracia digital: que democracia? In: MIGUEL, L. F.; BIROLI, F. (Org.). Mídia, representação e democracia. São Paulo: Hucitec, 2010. p. 241-259.

MENDONÇA, R. F.; AMARAL, E. F. L. Deliberação online em consultas públicas? O caso da Assembleia Legislativa de Minas Gerais. Revista Sociologia e Política, Paraná, v. 49, n. 22, p. 177203, 2014 .

MORAES, I. H. S. et al. Inclusão digital e conselheiros de saúde: uma política para a redução da desigualdade social no Brasil. Ciência e Saúde Coletiva, Rio de Janeiro, v. 14, n. 3, p. 879-888, 2009. TABUTEAU, D. Démocratie sanitaire: les nouveaux défis de la politique de santé. Paris: Odile Jacob, 2013.

\section{Contribuição dos autores}

Dallari e Aith conceberam e delinearam a pesquisa. Guerra, Silva, Falcão e Ventura fizeram a análise dos dados. Aith, Ventura, Guerra, Silva e Falcão escreveram o manuscrito. Dallari, Aith, Ventura, Balbinot e Bujdoso executaram a revisão crítica do manuscrito.

Recebido: 05/06/2016

Aprovado: 12/08/2016 